\title{
Long-Term Efficacy and Safety of Linagliptin in a Japanese Population with Type 2 Diabetes Aged $\geq 60$ Years Treated with Basal Insulin: A Randomised Trial
}

\author{
Eiichi Araki · Yuriko Unno (D) - Yuko Tanaka - Wataru Sakamoto • \\ Yuki Miyamoto
}

Received: January 28, 2019 / Published online: September 3, 2019

(c) The Author(s) 2019

\section{ABSTRACT}

Introduction: An estimated 4.3 million people aged $\geq 65$ years with diabetes live in Japan. We evaluated the efficacy and safety of linagliptin in older Japanese patients with poorly controlled type 2 diabetes (T2DM).

Methods: In this phase 4, randomised, placebocontrolled national study (part of a global study) conducted in Japan over a period of 52 weeks, 102 patients on stable treatment with basal insulin \pm metformin/alpha-glucosidase inhibitors were randomised (1:1) to receive linagliptin $5 \mathrm{mg}$ qd or placebo. The primary end point was the change in glycated haemoglobin

Enhanced Digital Features To view enhanced digital features for this article go to https://doi.org/10.6084/ m9.figshare.9576191.

Electronic Supplementary Material The online version of this article (https://doi.org/10.1007/s12325019-01065-7) contains supplementary material, which is available to authorized users.

E. Araki

Department of Metabolic Medicine, Kumamoto University Faculty of Life Sciences, Kumamoto, Japan

Y. Unno $(\bowtie) \cdot$ Y. Tanaka · W. Sakamoto ·

Y. Miyamoto

Medicine Division, Nippon Boehringer Ingelheim

Co. Ltd., Tokyo, Japan

e-mail: y.unno@r-and-o.jp
(HbA1c) after 24 weeks of treatment, with additional analyses at 52 weeks.

Results: Mean age and HbA1c of the study population were 71 years and $8.1 \%$, respectively. Approximately two-thirds of participants were aged $\geq 70$ years, two-thirds had macrovascular complications, approximately half had a baseline estimated glomerular filtration rate $<60 \mathrm{ml} / \mathrm{min} / 1.73 \mathrm{~m}^{2}$, and two-thirds had a time since diagnosis of diabetes $>10$ years. Significant HbA1c reductions with linagliptin vs. placebo were observed at 24 weeks, - $0.71 \%$ (95\% CI $-0.96,-0.45, p<0.0001)$, and maintained at 52 weeks, $-0.58 \%(95 \%$ CI -0.82 , $-0.34, p<0.0001)$. Linagliptin improved the chances of achieving a categorical HbA1c target $(<8.0 \%$ and $<7.0 \%)$ at 24 and 52 weeks in patients who were not at their respective target at the beginning of the study. Addition of linagliptin to insulin was associated with a numerical increase in the risk of any hypoglycaemia, but not in the risk of clinically significant hypoglycaemia, severe hypoglycaemia or recurring hypoglycaemia.

Conclusion: Linagliptin was effective in improving glucose control in Japanese patients aged $\geq 60$ years with T2DM on stable glucoselowering therapy with basal insulin. Linagliptin was well tolerated and no new safety concerns were raised. The results presented here are highly consistent with the results from the global study, which was conducted over a 24-week period. 
Trial Registration: ClinicalTrials.gov identifier, NCT02240680.

Funding: Boehringer Ingelheim and Eli Lilly and Company Diabetes Alliance.

Keywords: Basal insulin; DPP-4 inhibitors; Elderly patients; Japanese; Linagliptin; Type 2 diabetes

\section{INTRODUCTION}

Patients in East Asian countries with type 2 diabetes mellitus (T2DM) comprise approximately one-quarter of the global diabetes population [1]. Recent estimates indicate that 12 million people with diabetes live in Japan, of whom 4.3 million are aged $\geq 65$ years, placing Japan in the top six countries in the world with the largest absolute number of older people with diabetes [2]. Therefore, there is an imperative for treatments that provide safe and effective control of glycated haemoglobin (HbA1c) levels in these patients and that also reduce the risk of diabetes-related complications, which would place a huge burden on future healthcare resources.

It has been recognised since the 1970s that, compared with Caucasians, Japanese people with either normal or impaired glucose tolerance or with T2DM exhibit higher insulin sensitivity and reduced beta-cell function $[1,3,4]$. Such pathological differences in these populations have a major bearing on the best approach to T2DM treatment [4].

The Japan Diabetes Society (JDS) recommends an individualised approach for the treatment of T2DM; in the case of most patients the HbA1c target level is set to $<7.0 \%$, with the aim of preventing microvascular complications. However, when treatment intensification is difficult, as in the case of patients at high risk of hypoglycaemia, a less stringent target of HbA1c $<8.0 \%$ is recommended $[5,6]$. In elderly patients, the JDS and the Japan Geriatrics Society advise care when treatment with insulin, sulphonylureas (SUs) or glinides is used. Hypoglycaemia in the elderly is a particular concern of modern clinical care, and widely used therapies such as insulin and SUs have a known risk of hypoglycaemia. These medical societies advise that particular attention be paid to prevention of hypoglycaemia and recommend less stringent glycaemic targets in the case of patients with multiple comorbidities and reduced cognitive and physical function $[5,7]$.

Clinical practice guidelines for diabetes in Japan recommend insulin therapy as an option for patients with T2DM with inadequate glycaemic control despite diet, exercise and oral glucose-lowering therapy [8]. However, the precise risks of hypoglycaemia in the elderly, resulting from therapy combinations involving SUs and insulin specifically with dipeptidyl peptidase 4 (DPP-4) inhibitors-which are prescribed in $>70 \%$ of Japanese patients with T2DM [4] —have not been previously determined in dedicated clinical trials. Treatment combinations with insulin and various oral glucose-lowering drugs, including DPP-4 inhibitors, with their favourable safety profile and reduced risk of hypoglycaemia, could improve glycaemic control with a reduced dose of insulin [8] and, consequently, a lower risk of insulin-associated side effects.

DPP-4 inhibitors appear to have better glucose-lowering efficacy in Asian vs. non-Asian populations with T2DM. In a meta-analysis of 55 randomised, controlled trials, DPP- 4 inhibitors lowered HbA1c levels to a greater extent in studies comprising more than half of Asian patients vs. trials with less than half of Asian patients [9]. Dietary habits in East Asians may be a contributory factor, as studies show that the increased HbA1c-lowering effects of DPP-4 inhibitors correlated with intake of fish in patients with T2DM [4].

Over half of the patients with T2DM in Japan who initiate DPP-4 inhibitors are drug naïve [4]. A number of DPP-4 inhibitors are currently available in Japan, including linagliptin, which was launched in 2011. Linagliptin has been demonstrated to have a good safety profile and established efficacy in international clinical trials $[10,11]$. Consistent with these results, studies in Japanese populations confirmed the importance of linagliptin in the glucose-lowering drug armamentarium [12-14]. The prescribing information for linagliptin recommends reducing the dose of insulin and SUs when used in combination with linagliptin $[15,16]$. 
Studies undertaken with other DPP-4 inhibitors in the treatment of Japanese patients with T2DM show that these agents also exhibit good efficacy and safety profiles [17-23].

Older patients with a long-term history of diabetes often suffer from impaired renal function, which limits their therapy choices. Linagliptin has a predominantly non-kidney route of excretion and is one of the few DPP-4 inhibitors that do not require dose adjustments even in the case of impaired renal function [15]. A meta-analysis that included participants from phase 3 clinical trials showed that linagliptin was effective and well tolerated in patients with T2DM and chronic kidney disease (CKD) on background treatment with insulin [24].

While the clinical efficacy of linagliptin has been shown in Japanese patients with T2DM across a broad age range, the effects of linagliptin specifically in older Japanese patients have not been determined. We present here novel results of a dedicated phase 4 clinical trial, investigating the efficacy, safety and tolerability of linagliptin, added as intensification of glucose-lowering therapy (i.e., according to investigator judgement of the need to improve glycaemic control based on individualised HbA1c targets), in older individuals from Japan, with insufficiently controlled T2DM while on stable background treatment with basal insulin.

\section{METHODS}

\section{Study Overview}

This local study conducted in Japan was part of a global study (Clinical Trials.gov: NCT02240680) investigating the efficacy and safety of linagliptin in older patients on stable treatment with insulin [25]. The global study was conducted over a 24-week period, while the study in Japanese participants was extended to 52 weeks.

\section{Ethics}

The trial protocol was reviewed by the Independent Ethics Committees and/or
Institutional Review Boards of the trial participating study sites. The study was conducted in accordance with the principles of the Declaration of Helsinki, the ICH GCP, the Japanese GCP regulations and Good Post-Marketing Study Practice. All patients provided written informed consent.

\section{Inclusion Criteria}

Male and female Japanese patients aged $\geq 60$ years with a clinical diagnosis of T2DM treated with basal insulin maintained at a stable dose for $\geq 4$ weeks prior to randomisation, with HbA1c levels of $7.0-10.0 \%$ and a body mass index $\leq 45 \mathrm{~kg} / \mathrm{m}^{2}$, were included in the study. Permitted insulin formulations included long- and intermediate-acting insulins only. The only permitted oral glucose-lowering drugs were metformin and/or alpha-glucosidase inhibitors (used at a stable dose for $\geq 12$ weeks prior to randomisation).

\section{Exclusion Criteria}

Individuals could not be enrolled if they were being treated with any glucose-lowering therapies not included in the permitted list (SUs, thiazolidinediones, meglitinides, bromocriptine, sodium glucose cotransporter-2 inhibitors, DPP-4 inhibitors or glucagon-like peptide-1 analogues) or any anti-obesity medication, were depressed (assessed using the Patient Health Questionnaire) or cognitively impaired (assessed using the Saint Louis University Mental Status Examination), or had acute coronary syndrome, indication of liver disease, history of cancer or bariatric surgery.

\section{Study Design}

Following screening and a 1-week placebo runin, participants were randomised to receive either linagliptin $5 \mathrm{mg}$ qd or placebo for 52 weeks. For the global study, a sample size of 300 patients ( 150 per study arm) was calculated to provide at least $99 \%$ power at the two-sided significance level of 5\% to show superiority for HbA1c reduction from baseline [25]. In Japan, 
the randomisation of at least 100 patients was planned; a proportion of 33\% (Japanese population vs. total population of the global study) was considered sufficient to assess consistency of the results of the primary end point between the Japanese sub-population and total population according to the MHLW guidance 'Basic principles on Global Clinical Trials' [26].

The randomisation, study procedures and statistical analysis have previously been reported for the global study [25]. Briefly, patients were randomly assigned to one of the two treatment arms; linagliptin $5 \mathrm{mg}$ qd or placebo, in a 1:1 ratio. Randomisation was stratified based on an HbA1c at screening $(<8.5 \%$ vs. $\geq 8.5 \%)$ and insulin dose at the beginning of the run-in period ( $<40 \mathrm{IU}$ vs. $\geq 40 \mathrm{IU})$. Study visits were scheduled at randomisation and at the end of weeks $6,12,18,24,25,30,36,42,48$ and 52. Rescue medication was initiated if increasing the permitted glucose-lowering therapy was unsuccessful in reducing fasting plasma glucose (FPG) to $\leq 270 \mathrm{mg} / \mathrm{dl}$ after 6 weeks. DPP-4 inhibitors and glucagon-like peptide 1 receptor agonists were not permitted as rescue medication. After week 24, the permitted glucose-lowering therapy could be increased or adjusted at the discretion of the investigator even if the above criteria were not met, when the investigator considered such an adjustment to be optimal for the patient's welfare.

All adverse events (AEs) were classified based on the Medical Dictionary for Regulatory Activities (MedDRA), version 20.0. Adverse events of special interest (AESI) were: hypersensitivity reactions (such as angioedema, angioedema-like events and anaphylaxis), skin lesions (such as exfoliative rash, skin necrosis or bullous dermatitis), hepatic events [such as $\geq 3$ fold upper limit of normal (ULN) of aspartate aminotransferase and/or alanine aminotransferase in combination with an elevation of total bilirubin > twofold ULN measured in the same blood draw sample], renal adverse events (such as acute renal failure), pancreatitis and pancreatic cancer.

\section{End Points and Statistical Analysis}

The primary end point was defined as the change in HbA1c from baseline after 24 weeks of treatment. Secondary end points were the proportion of patients achieving HbA1c targets of $<7.0 \%$ and $<8.0 \%$ after 24 and 52 weeks, the proportion of patients experiencing $\geq 1$ hypoglycaemic event accompanied by a prespecified glucose value during 24 and 52 weeks, the change from baseline in HbA1c and FPG at $6,12,18,30,36,42,48$ and 52 weeks, and change from baseline in FPG at 24 weeks, the incidence rate of hypoglycaemia accompanied by a prespecified glucose value $>24$ and 52 weeks, the proportion of patients with HbA1c $<7.0 \%$ and $<8.0 \%$ and no hypoglycaemia accompanied by a prespecified glucose value after 24 and 52 weeks, and the basal insulin dose change over 24 and 52 weeks.

Hyperglycaemia and hypoglycaemia were reviewed based on data collected from selfmonitoring blood glucose (SMBG) devices and on-site FPG values. Investigators could, at their discretion, advise lifestyle modifications or therapy adjustments consisting of dose changes of the permitted drugs-the criteria for adjusting the background insulin therapy dose or permitted glucose-lowering drugs (metformin or alpha-glucosidase inhibitors) were FPG $>270 \mathrm{mg} / \mathrm{dl}$ during weeks 0-12, FPG $>240 \mathrm{mg} / \mathrm{dl}$ during weeks 12-24 and FPG $>180 \mathrm{mg} / \mathrm{dl}$ after week 24 .

Hypoglycaemia accompanied by a prespecified glucose value was defined as any investigator- reported hypoglycaemia with blood glucose $(\mathrm{BG})<54 \mathrm{mg} / \mathrm{dl}(<3.0 \mathrm{mmol} / \mathrm{l})$ or any symptomatic hypoglycaemic event with $\mathrm{BG} \leq 70 \mathrm{mg} / \mathrm{dl}(\leq 3.9 \mathrm{mmol} / \mathrm{l})$ or any severe hypoglycaemic event (defined as requiring third-party assistance to administer carbohydrate or glucagon). Clinically significant hypoglycaemia has been recently defined in the ADA statement as glycaemia with a BG value $<54 \mathrm{mg} / \mathrm{dl}$ [27]. BG was measured by the central laboratory (FPG) or SMBG device.

The changes from baseline in HbA1c and FPG were analysed using mixed models for repeated measurements (MMRM) using the full analysis set (FAS; defined as all randomised 
patients who received one dose of study drug and had a baseline and $\geq 1$ on-treatment HbA1c value), using observed cases (OC). For HbA1c the model included treatment, baseline HbA1c, baseline insulin dose, week, week by baseline HbA1c interaction and week by treatment interaction. For FPG the model included treatment, baseline $\mathrm{HbA1c}$, baseline FPG, baseline insulin dose, week, week by baseline FPG interaction and week by treatment interaction.

A logistic regression was conducted (FAS, OC) for the proportion of patients who experienced at least one hypoglycaemia event during 24 or 52 weeks. The model included treatment as a fixed effect with baseline HbA1c and baseline daily basal insulin dose as linear covariates. The proportion of patients with HbA1c $<7.0 \%$ and $<8.0 \%$ after 24 and 52 weeks of treatment were analysed on the FAS, using the appoach where non-completers are considered failure (NCF; patients who do not complete 24 and 52 weeks of treatment are considered non-responders), by determining the percentage of patients that fulfilled the responder criteria by treatment. A logistic regression model with treatment as fixed effect and baseline HbA1c and baseline daily basal insulin dose as linear covariates was applied.

Safety end points were analysed descriptively; standard safety analyses were performed based on the treated set (TS), which comprised all patients treated with at least one dose of randomised study medication. The safety analyses were based on data collected up to 52 weeks; any AEs occurring within 7 days of last study medication (residual period) were considered on-treatment. All AEs up to and including day 175 were included in the 24 -week analysis; for the patients that prematurely discontinued prior to or at day 175 , an additional residual period of 7 days was added.

\section{RESULTS}

\section{Patients}

Out of 108 participants screened, 102 were randomised 1:1 to linagliptin and placebo (52 and 50 patients, respectively). During the study period, seven participants in the linagliptin group and eight in the placebo group discontinued the trial (Supplementary Fig. 1). Approximately two-thirds of the participants were aged $\geq 70$ years, confirming the relevance of the study for an elderly population (Table 1). In addition, over two-thirds of the participants had a history of diabetes $>10$ years, while $<10 \%$ had a history of diabetes of $<5$ years at the beginning of the study (Table 1). Approximately half of the participants had a baseline estimated glomerular filtration rate (eGFR) $<60 \mathrm{ml} / \mathrm{min} /$ $1.73 \mathrm{~m}^{2}$, and with one exception all participants had a baseline eGFR $<90 \mathrm{ml} / \mathrm{min} / 1.73 \mathrm{~m}^{2}$ (Table 1).

Microvascular disease, hypertension and hyperlipidaemia were highly prevalent in this trial population, each being diagnosed at baseline in two-thirds of the participants or more. Conversely, complications associated with advanced stages of T2DM, such as macrovascular disease (excluding hypertension) and diabetic foot, had lower prevalence rates (Supplementary Table 1).

More than $95 \%$ of the participants were treated with long-acting insulins, while over half of the participants were treated at baseline with oral glucose-lowering drugs (metformin and alpha-glucosidase inhibitors) in addition to insulin (Supplementary Table 2).

\section{Efficacy}

The treatment difference in HbA1c reductions for linagliptin vs. placebo was $-0.71 \%$ at 24 weeks [95\% confidence interval (CI) -0.96 , $-0.45, p<0.0001]$ and $-0.58 \%$ at 52 weeks (95\% CI $-0.82,-0.34, p<0.0001)$, as shown in Fig. 1a, b. The corresponding difference in FPG reductions for linagliptin vs. placebo was $-15.0 \mathrm{mg} / \mathrm{dl}$ at 24 weeks $(95 \% \mathrm{CI}-26.6,-3.3$, $p=0.0123)$ and $-3.4 \mathrm{mg} / \mathrm{dl}$ at 52 weeks $(95 \%$ CI $-15.8,9.1, p=0.5916)$, as shown in Fig. 1c.

The treatment effect on HbA1c levels was consistent across subgroups, the point estimate for the overall Japanese population being within the confidence intervals of all subgroups analysed. An interaction $p$ value $<0.10$ was observed only for age and gender categories 
Table 1 Baseline demographics and clinical characteristics

\begin{tabular}{|c|c|c|}
\hline & Linagliptin & Placebo \\
\hline Patients (treated set ${ }^{\mathrm{a}}$ ), $N$ & 52 & 50 \\
\hline Male & $34(65.4)$ & $36(72.0)$ \\
\hline Age, years, mean (SD) & $71.1(5.5)$ & $71.5(5.6)$ \\
\hline$\geq 60$ to $<65$ years, $n(\%)$ & $7(13.5)$ & $4(8.0)$ \\
\hline$\geq 65$ to $<70$ years, $n(\%)$ & $10(19.2)$ & $14(28.0)$ \\
\hline$\geq 70$ to $<75$ years, $n(\%)$ & $24(46.2)$ & $19(38.0)$ \\
\hline$\geq 75$ years, $n(\%)$ & $11(21.2)$ & $13(26.0)$ \\
\hline BMI, $\mathrm{kg} / \mathrm{m}^{-2}$, mean $(\mathrm{SD})$ & $23.9(3.3)$ & $22.9(3.0)$ \\
\hline $\begin{array}{l}\text { eGFR (MDRD), ml/min/ } \\
1.73 \mathrm{~m}^{2}, \text { mean }(\mathrm{SD})\end{array}$ & $59.9(14.0)$ & $62.7(11.1)$ \\
\hline $\begin{array}{l}\geq 90 \mathrm{ml} / \mathrm{min} / 1.73 \mathrm{~m}^{2}, \\
n(\%)\end{array}$ & $1(1.9)$ & $0(0.0)$ \\
\hline $\begin{array}{l}\geq 60 \text { to }<90 \mathrm{ml} / \mathrm{min} / \\
1.73 \mathrm{~m}^{2}, n(\%)\end{array}$ & $22(42.3)$ & $31(62.0)$ \\
\hline $\begin{array}{l}\geq 45 \text { to }<60 \mathrm{ml} / \mathrm{min} / \\
1.73 \mathrm{~m}^{2}, n(\%)\end{array}$ & $22(42.3)$ & $16(32.0)$ \\
\hline $\begin{array}{l}\geq 30 \text { to }<45 \mathrm{ml} / \mathrm{min} / \\
1.73 \mathrm{~m}^{2}, n(\%)\end{array}$ & $7(13.5)$ & $3(6.0)$ \\
\hline Patients (full analysis set ${ }^{\mathrm{b}}$ ), $N$ & 51 & 50 \\
\hline HbAlc, \%, mean (SD) & $8.1(0.8)$ & $8.0(0.7)$ \\
\hline$<7.0, n(\%)$ & $2(3.9)$ & $1(2.0)$ \\
\hline$\geq 7.0$ to $<7.5, n(\%)$ & $10(19.6)$ & $9(18.0)$ \\
\hline$\geq 7.5$ to $<8.0, n(\%)$ & $14(27.5)$ & $14(28.0)$ \\
\hline$\geq 8.0$ to $<9.0, n(\%)$ & $17(33.3)$ & $22(44.0)$ \\
\hline$\geq 9.0, n(\%)$ & $8(15.7)$ & $4(8.0)$ \\
\hline FPG, mg/dl, mean (SD) & $130.8(31.4)$ & $127.6(37.6)$ \\
\hline \multicolumn{3}{|c|}{ Time since diagnosis of diabetes, $n(\%)$} \\
\hline$\leq 1$ year & $2(3.9)$ & $0(0.0)$ \\
\hline$>1$ to $\leq 5$ years & $4(7.8)$ & $3(6.0)$ \\
\hline$>5$ to $\leq 10$ years & $8(15.7)$ & $13(26.0)$ \\
\hline
\end{tabular}

Table 1 continued

\begin{tabular}{lll}
\hline & Linagliptin & Placebo \\
\hline$>10$ to $\leq 15$ years & $13(25.5)$ & $12(24.0)$ \\
$>15$ years & $24(47.1)$ & $22(44.0)$ \\
\hline
\end{tabular}

$B M I$ body mass index, $e G F R$ estimated glomerular filtration rate, FPG fasting plasma glucose, $H b A l c$ glycated haemoglobin, $S D$ standard deviation

a All patients who were treated with $\geq 1$ dose of study medication

b All patients who were treated with $\geq 1$ dose of study medication and had a baseline and $\geq 1$ on-treatment HbAlc measurement

(Supplementary Fig. 2). There was an increase in the chance of achieving a categorical HbA1c target of $<7.0 \%$ (in patients with baseline HbA1c $\geq 7.0 \%$ ) and $<8.0 \%$ (in patients with baseline $\mathrm{HbA1c} \geq 8.0 \%$ ), as shown in Supplementary Fig. 3. Similarly, a trend was observed towards improved chances of achieving categorical HbA1c targets without hypoglycaemia accompanied by a prespecified glucose value (Fig. 2).

During the study, more patients in the placebo group had their insulin dose increased compared with patients in the linagliptin group. Conversely, more patients receiving linagliptin vs. placebo had their insulin dose reduced during the study. These trends were consistent at 24 and 52 weeks (Supplementary

Fig. 1 Change in HbAlc from baseline at weeks 24 and 52 (a); change over time in HbAlc (b) and FPG (c). ${ }^{a}$ MMRM analysis, FAS (OC), model includes baseline $\mathrm{HbA1c}$, baseline daily basal insulin, baseline HbAlc by week interaction as linear covariates, treatment, week, treatment by week interaction as fixed effects and patient as random effect. ${ }^{b}$ MMRM analysis, FAS (OC), model includes treatment, week and treatment by week interaction as fixed categorical effects and baseline FPG, baseline $\mathrm{HbAlc}$ and baseline daily basal insulin dose as fixed linear covariates as well as baseline FPG by week interaction. CI confidence interval, FAS full analysis set-all patients who were treated with $\geq 1$ dose of study medication and had a baseline and $\geq 1$ on-treatment HbAlc measurement. HbAlc glycated haemoglobin, MMRM mixed model repeating measurements, $O C$ observed cases 
(a)
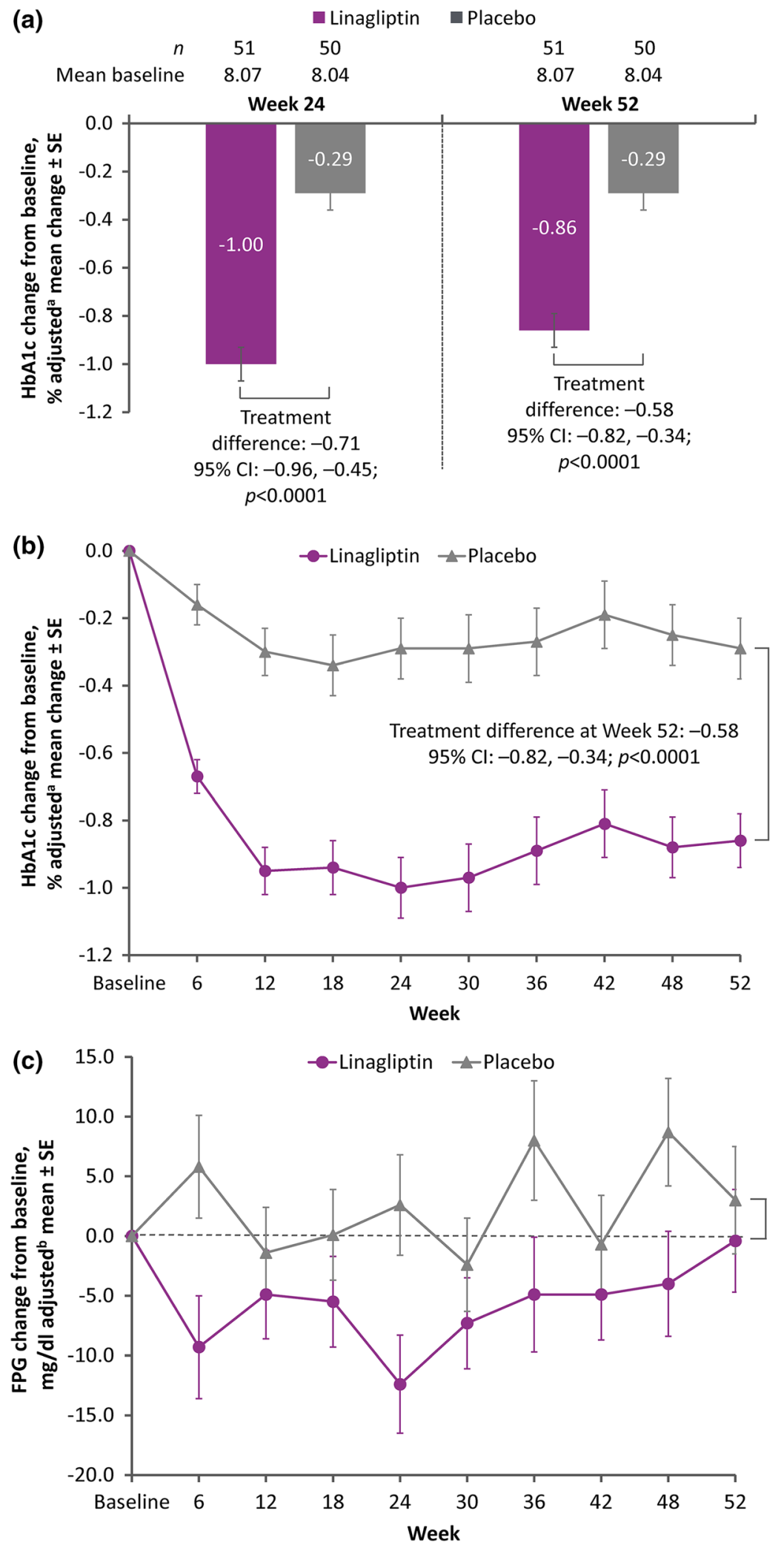

Treatment difference at Week 52: -3.4 $95 \% \mathrm{Cl}$ : $-15.8,9.1$ $p=0.5916$ 
(a)

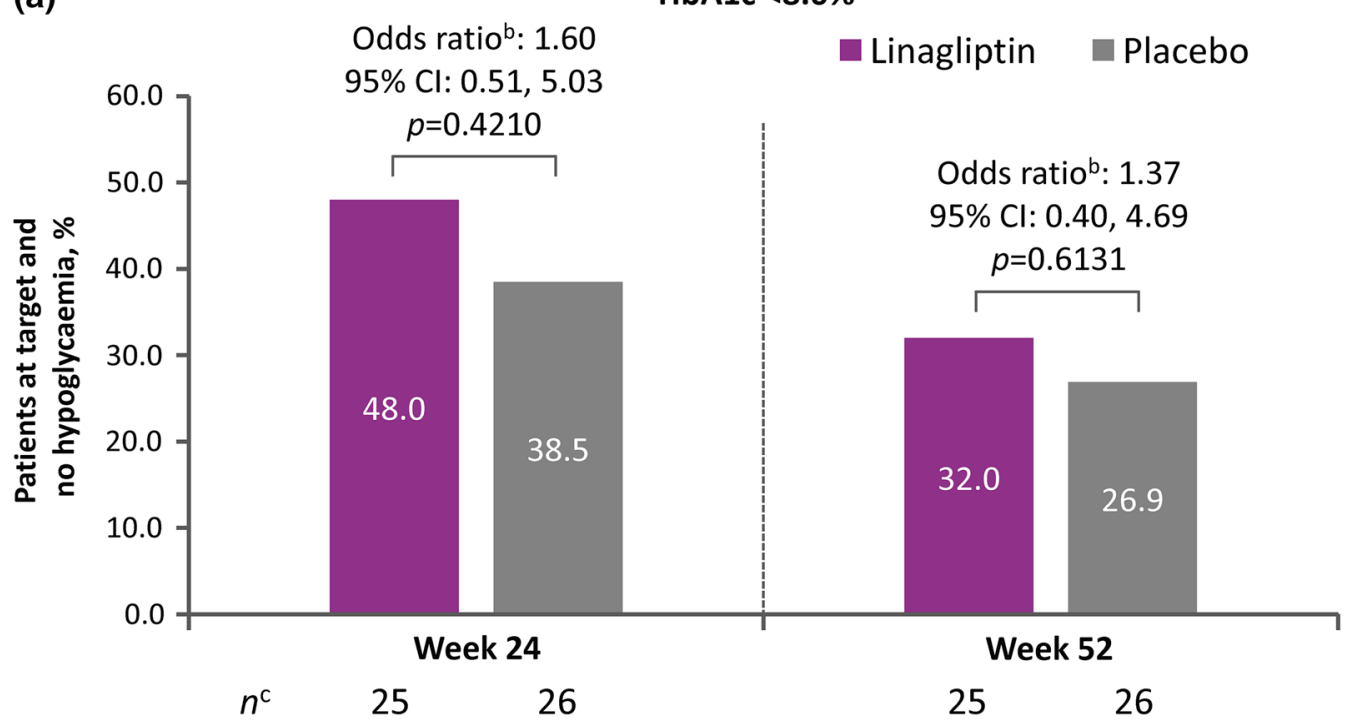

(b)

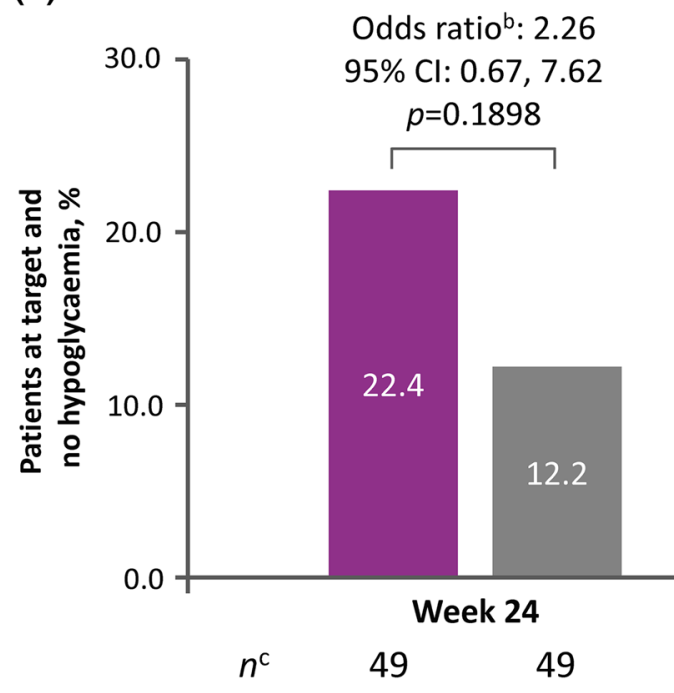

Fig. 2 Proportion of patients with $\mathrm{HbAlc}<8.0 \%$ (a) and $<7.0 \%$ (b) and no hypoglycaemia accompanied by a prespecified glucose value a after 24 and 52 weeks, FAS (NCF). ${ }^{a}$ Any hypoglycaemia with $\mathrm{BG}<54 \mathrm{mg} / \mathrm{dl}$ $(3.0 \mathrm{mmol} / \mathrm{l})$ as measured by the central laboratory (FPG) or SMBG device, any symptomatic event $\leq 70 \mathrm{mg} / \mathrm{dl}(3.9 \mathrm{mmol} / \mathrm{l})$ or severe hypoglycaemia (requiring third-party assistance to administer carbohydrate or glucagon). ${ }^{b}$ Logistic regression model includes continuous baseline HbAlc and baseline daily basal insulin

Table 3) and mirror the results from the global study [25].

The proportion of patients experiencing $\geq 1$ hypoglycaemic event accompanied by a
$\mathrm{HbA1c}<7.0 \%$
HbA1c $<8.0 \%$
- Linagliptin Placebo

Odds ratio ${ }^{\text {b }} 1.37$

\% Cl: $0.40,4.69$

25
Odds ratio ${ }^{b}: 3.20$

95\% Cl: 0.76, 13.54

$p=0.1147$

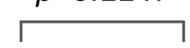

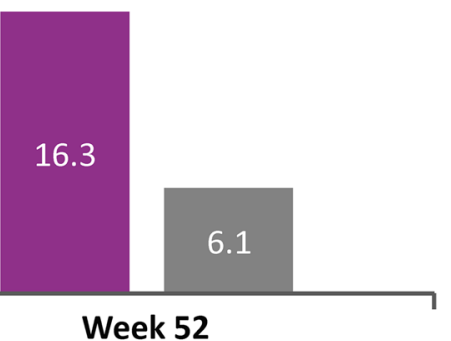

49

49 as linear covariates and treatment as a fixed effect. ${ }^{c}$ Only participants who were not at the HbAlc target of $<8.0 \%$ and $<7.0 \%$ at the beginning of the trial (HbAlc $\geq 8.0 \%$ and $\geq 7.0 \%$, respectively) were included in the analysis. $C I$ confidence interval, FAS full analysis set-all patients who were treated with $\geq 1$ dose of study medication and had a baseline and $\geq 1$ on-treatment HbAlc measurement. FPG fasting plasma glucose, $H b A 1 c$ glycated haemoglobin, $N C F$ non-completers considered failures, $S M B G$ self-monitoring blood glucose

prespecified glucose value during 24 or 52 weeks was numerically higher in patients treated with linagliptin vs. placebo, but this difference was not statistically significant (Fig. 3a). In addition, 

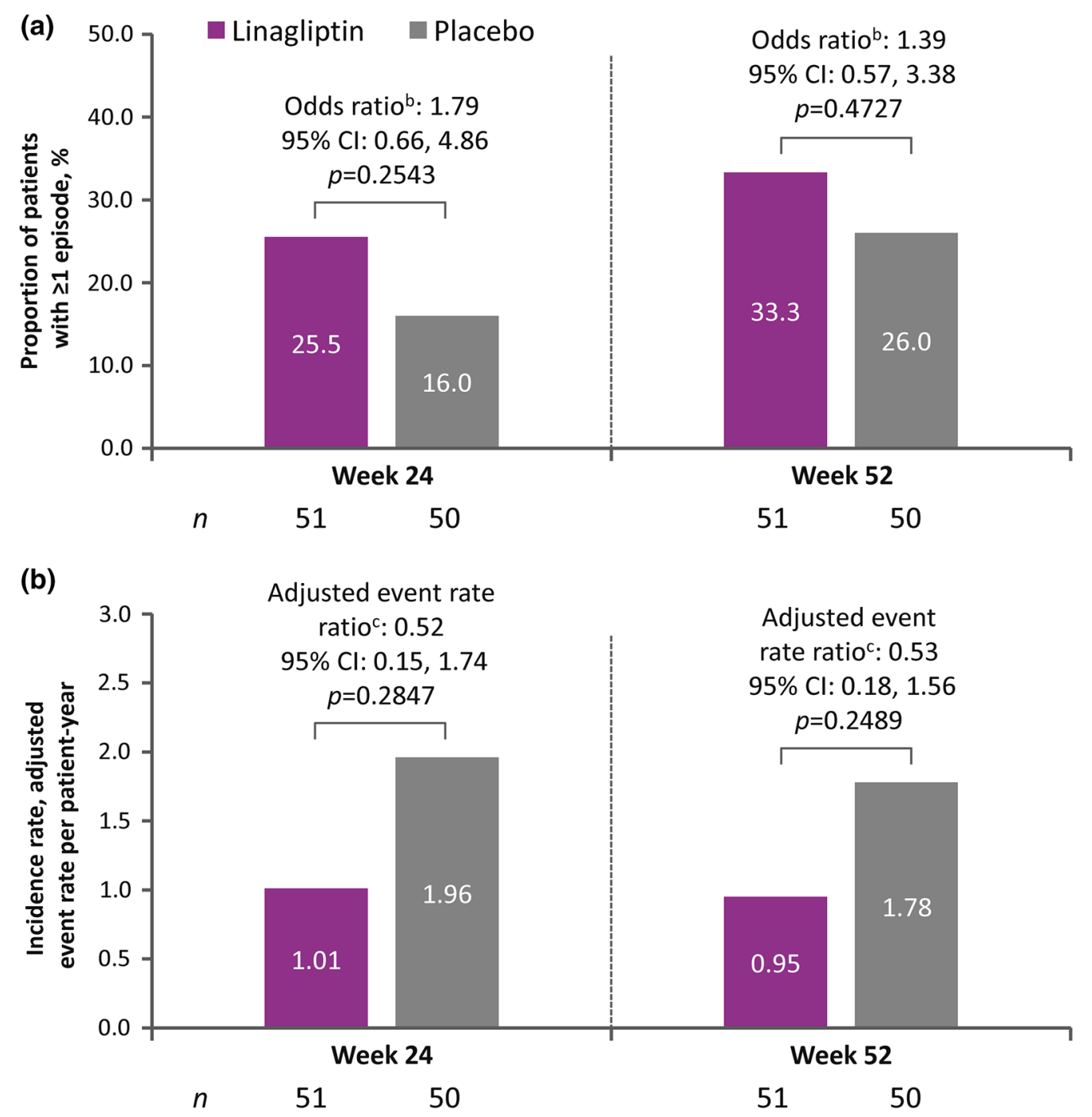

Fig. 3 Hypoglycaemia accompanied by a prespecified glucose value ${ }^{a}$ over 24 and 52 weeks, FAS (OC): proportion of patients with $\geq 1$ episode $(\mathbf{a})$ and recurring hypoglycaemia (b). ${ }^{a}$ Any hypoglycaemia with BG $<54 \mathrm{mg} / \mathrm{dl}(3.0 \mathrm{mmol} / \mathrm{l})$ as measured by the central laboratory (FPG) or SMBG device, or any symptomatic hypoglycaemic event with $B G \leq 70 \mathrm{mg} / \mathrm{dl}(3.9 \mathrm{mmol} / \mathrm{l})$ or any severe hypoglycaemic event (defined as requiring third-party assistance to administer carbohydrate or glucagon). ${ }^{b}$ Logistic regression model includes continuous

a numerical, non-significant decrease was observed in the incidence of recurring hypoglycaemia accompanied by a prespecified glucose level with linagliptin compared with placebo (Fig. 3b). Moreover, at week 52 linagliptin treatment compared with placebo was associated baseline HbAlc, baseline daily basal insulin as linear covariates and treatment as a fixed effect. "Negative binomial model includes terms for treatment, baseline $\mathrm{HbAlc}$, baseline daily basal insulin dose and adjusted for $\log$ (days of follow-up). $B G$ blood glucose, $C I$ confidence interval, $F A S$ full analysis set-all patients who were treated with $\geq 1$ dose of study medication and had a baseline and $\geq 1$ on-treatment HbAlc measurement. FPG fasting plasma glucose, $O C$ observed cases, $S M B G$ selfmonitoring blood glucose

with a numerical decrease in both the frequency and incidence rate of clinically significant hypoglycaemia (assessed in accordance with the ADA statement) [27]: hypoglycaemia with a BG value $<54 \mathrm{mg} / \mathrm{ml}(3.0 \mathrm{mmol} / \mathrm{l})$ or severe hypoglycaemia (Supplementary Fig. 4). 


\section{Safety}

Linagliptin as addition to insulin was well tolerated in the current study, which is consistent with findings from previously published studies. The incidence of severe AEs and AEs leading to discontinuation of therapy was low (Table 2). Investigator-defined drug-related AEs were numerically higher in the linagliptin group, and this treatment difference was driven entirely by hypoglycaemia (placebo: 18.0\%, linagliptin: $26.9 \%$ ), which was the only drug-related $\mathrm{AE}$ reported in the linagliptin group (Table 2). The incidence of any AEs was numerically higher in the linagliptin group, with the most common AEs being hypoglycaemia in both groups and the common cold (viral upper respiratory tract infection) in the linagliptin group (Table 2). The incidence of AESI was low and there were no reported occurrences of skin lesions, acute pancreatitis or pancreatic cancer (Table 2).

An increase in the risk of any hypoglycaemic AE was observed with linagliptin vs. placebo (Supplementary Table 4). However, this imbalance was mainly due to mild, asymptomatic hypoglycaemia, as there was no clinically meaningful difference between linagliptin vs. placebo in the proportion of patients with clinically important hypoglycaemia (blood glucose $<54 \mathrm{mg} / \mathrm{dl}$ or severe hypoglycaemia; Supplementary Table 4). In the case of severe hypoglycaemia, the incidence did not exceed $2.0 \%$ for treatment at either week 24 or 52 . Furthermore, there was no increase in the risk of recurrent episodes of any hypoglycaemia ( 2 or more, for the same patient; Supplementary Table 4).

\section{DISCUSSION}

We report here the results of a phase 4 clinical study conducted in Japan over a 52-week period, which investigated the efficacy and safety of linagliptin compared with placebo in older patients with T2DM and inadequate glycaemic control while on stable background therapy with insulin with or without additional glucoselowering drugs. Linagliptin was effective in improving the glycaemic control in this trial population, without increasing the risk of clinically significant hypoglycaemia.

In terms of glycaemic control and risk of hypoglycaemia, the results from the Japanese study at 24 and 52 weeks were consistent with the results from the global study, which was only conducted for 24 weeks [25]. In line with the results from the global study, linagliptin vs. placebo was effective in helping participants in the Japanese study population to achieve significantly lower levels of HbA1c; this finding was confirmed at both 24 and 52 weeks. In addition, more patients treated with linagliptin compared with placebo achieved categorical HbA1c targets and categorical HbA1c targets without hypoglycaemia; similar results were observed in the global study [25].

Subgroup analysis indicated that the efficacy of linagliptin was generally consistent across subcategories. Nevertheless, no definite conclusions should be drawn from these data, considering the low number of patients in each subgroup. In the global study, the treatment effect was consistent across age subcategories in respect to HbA1c changes achieved [25].

The data presented here provide an indication that the addition of linagliptin might help reduce the required dose of basal insulin, thus alleviating the risk of hypoglycaemia. However, as the number of patients with modified insulin dose throughout the course of this trial was too small to provide sufficient statistical power, future studies are warranted to confirm the effects of linagliptin in a larger cohort of older Japanese patients and investigate this aspect in more detail.

In the current study, there was no increase in the risk of clinically significant hypoglycaemia (hypoglycaemia with a glucose value $<54 \mathrm{mg}$ / $\mathrm{dl}$ or hypoglycaemia requiring third-party assistance) and no increase in the incidence of recurring hypoglycaemia with linagliptin vs. placebo. However, there was a trend for a higher risk of any hypoglycaemia, mainly driven by asymptomatic hypoglycaemia, associated with linagliptin. These results mirror the observations from the global study [25].

Linagliptin is a glucose-lowering drug with a good safety profile established across multiple clinical trials [11]. Meta-analyses that included 
Table 2 Summary of adverse events

\begin{tabular}{|c|c|c|c|c|}
\hline & \multicolumn{2}{|l|}{24 weeks } & \multicolumn{2}{|l|}{52 weeks } \\
\hline & Linagliptin & Placebo & Linagliptin & Placebo \\
\hline Patients (treated set ${ }^{a}$ ) & 52 & 50 & 52 & 50 \\
\hline Any AE & $35(67.3)$ & $27(54.0)$ & $47(90.4)$ & $38(76.0)$ \\
\hline Severe AE & $4(7.7)$ & $3(6.0)$ & $8(15.4)$ & $5(10.0)$ \\
\hline Drug-related AE (investigator-defined) & $14(26.9)$ & $4(8.0)$ & $14(26.9)$ & $10(20.0)$ \\
\hline AE leading to discontinuation & $4(7.7)$ & $3(6.0)$ & $7(13.5)$ & $5(10.0)$ \\
\hline Serious $A E^{b}$ & $8(15.4)$ & $5(10.0)$ & $13(25.0)$ & $7(14.0)$ \\
\hline AEs with $>2 \%$ incidence & $35(67.3)$ & $27(54.0)$ & $47(90.4)$ & $38(76.0)$ \\
\hline Ear and labyrinth disorders & $0(0.0)$ & $0(0.0)$ & $2(3.8)$ & $0(0.0)$ \\
\hline Eye disorders & $4(7.7)$ & $0(0.0)$ & $5(9.6)$ & $2(4.0)$ \\
\hline Gastrointestinal disorders & $9(17.3)$ & $1(2.0)$ & $11(21.2)$ & $2(4.0)$ \\
\hline Infections and infestations & $11(21.2)$ & $4(8.0)$ & $22(42.3)$ & $9(18.0)$ \\
\hline Investigations & $1(1.9)$ & $3(6.0)$ & $3(5.8)$ & $5(10.0)$ \\
\hline Metabolism and nutrition disorders & $17(32.7)$ & $9(18.0)$ & $21(40.4)$ & $16(32.0)$ \\
\hline Diabetes mellitus & $0(0.0)$ & $1(2.0)$ & $0(0.0)$ & $2(4.0)$ \\
\hline Hypoglycaemia & $16(30.8)$ & $8(16.0)$ & $20(38.5)$ & $14(28.0)$ \\
\hline Musculoskeletal and connective tissue disorders & $2(3.8)$ & $6(12.0)$ & $8(15.4)$ & $8(16.0)$ \\
\hline Reproductive system and breast disorders & $3(5.8)$ & $0(0.0)$ & $3(5.8)$ & $0(0.0)$ \\
\hline Skin and subcutaneous tissue disorders & $4(7.7)$ & $3(6.0)$ & $7(13.5)$ & $5(10.0)$ \\
\hline Vascular disorders & $0(0.0)$ & $1(2.0)$ & $2(3.8)$ & $1(2.0)$ \\
\hline Hypertension & $0(0.0)$ & $1(2.0)$ & $2(3.8)$ & $1(2.0)$ \\
\hline \multicolumn{5}{|l|}{ AEs of special interest } \\
\hline Hypersensitivity reactions & $3(5.8)$ & $1(2.0)$ & $5(9.6)$ & $1(2.0)$ \\
\hline Dermatitis allergic & $0(0.0)$ & $0(0.0)$ & $1(1.9)$ & $0(0.0)$ \\
\hline Dermatitis contact & $2(3.8)$ & $0(0.0)$ & $2(3.8)$ & $0(0.0)$ \\
\hline Eczema & $0(0.0)$ & $0(0.0)$ & $2(3.8)$ & $0(0.0)$ \\
\hline Rash & $0(0.0)$ & $1(2.0)$ & $0(0.0)$ & $1(2.0)$ \\
\hline Urticaria & $1(1.9)$ & $0(0.0)$ & $1(1.9)$ & $0(0.0)$ \\
\hline Skin lesions & $0(0.0)$ & $0(0.0)$ & $0(0.0)$ & $0(0.0)$ \\
\hline Hepatic events & $1(1.9)$ & $0(0.0)$ & $2(3.8)$ & $1(2.0)$ \\
\hline Alanine aminotransferase increased & $0(0.0)$ & $0(0.0)$ & $1(1.9)$ & $1(2.0)$ \\
\hline Hepatic steatosis & $1(1.9)$ & $0(0.0)$ & $1(1.9)$ & $0(0.0)$ \\
\hline Renal AEs & $1(1.9)$ & $0(0.0)$ & $1(1.9)$ & $0(0.0)$ \\
\hline
\end{tabular}


Table 2 continued

\begin{tabular}{|c|c|c|c|c|}
\hline & \multicolumn{2}{|l|}{24 weeks } & \multicolumn{2}{|l|}{52 weeks } \\
\hline & Linagliptin & Placebo & Linagliptin & Placebo \\
\hline Renal impairment & $1(1.9)$ & $0(0.0)$ & $1(1.9)$ & $0(0.0)$ \\
\hline Pancreatitis & $0(0.0)$ & $0(0.0)$ & $0(0.0)$ & $0(0.0)$ \\
\hline Pancreatic cancer & $0(0.0)$ & $0(0.0)$ & $0(0.0)$ & $0(0.0)$ \\
\hline
\end{tabular}

$A E$ adverse event

a All patients who were treated with $\geq 1$ dose of study medication

b A patient may be counted in $>1$ seriousness criterion

data from participants aged $\geq 65$ years have confirmed that the safety profile of linagliptin also extends to its use in the elderly population $[28,29]$. In the current study conducted in Japanese older patients, linagliptin was well tolerated, and AEs occurred with similar rates in both the linagliptin and placebo groups. In particular, the incidence of severe AEs was low and AESI occurred at the expected rate. No new safety concerns were reported during the 52 weeks of treatment. Overall, the safety results observed in this study were consistent with those observed in the global study [25] and with the expected results based on previous clinical trials for linagliptin.

Other DPP-4 inhibitors have been evaluated in the treatment of Japanese patients with T2DM and, like linagliptin, have demonstrated favourable efficacy and safety profiles [17-23]. In the majority of these studies, the mean age was lower (approximately 60 years) than in the current study (71 years). However, in a recent study by Fukuda et al. [21], the efficacy and safety of sitagliptin were assessed in a 52-week, observational, single-arm study comprising a larger $(N=5130)$ cohort of the elderly Japanese population (mean age $73.8 \pm 6.1$ years), with a focus on hypoglycaemia. Over 52 weeks, sitagliptin resulted in a significant reduction from baseline in HbA1c $(-0.7 \pm 1.1 \%, p<0.001)$, with no increase in the percentage of patients with hypoglycaemia. However, hypoglycaemia occurred more frequently with add-on therapy to SU or when the dose of sitagliptin was increased in combination therapy and, from this, the authors concluded that sitagliptin should be used with caution [21].
The risk of infections is an important consideration in the context of diabetes, especially in the case of elderly patients or those with associated comorbidities. Several reports, including some for linagliptin, have mentioned a higher risk of infectious AEs with DPP-4 inhibitors [12, 30-33]. Moreover, the Japanese label information for linagliptin lists nasopharyngitis as an adverse drug reaction reported in $>0.3 \%$ of the patients [16]. In the global study, the infection and infestation AE prevalence was slightly higher with linagliptin vs. placebo; these occurred in 34 patients $(22.5 \%)$ in the placebo group and 38 patients $(25.2 \%)$ in the linagliptin group [25]. Overall, no new safety finding can be reported based on the data from the current study for these types of AEs.

The key strength of the current study was that it was designed to provide clear and specific data on the use of linagliptin in an older Japanese population over a 52-week period. The consequent limitation is that the results may not be directly extrapolated to other Asian groups. Furthermore, our analyses of hypoglycaemia can only be considered exploratory: due to recruitment difficulties, the initially planned total number of participants was lowered through a protocol amendment; thus, the assessment of hypoglycaemia was no longer statistically powered.

In a protocol amendment, to further facilitate recruitment, the minimum age inclusion criterion was lowered to 60 years in the global study; nevertheless, in the Japanese trial, twothirds of the participants were aged $\geq 70$ years; consequently, our study results are highly relevant for an elderly population. 


\section{CONCLUSIONS}

The results of the present study in Japanese patients with T2DM conducted over 52 weeks were highly consistent with the results of the 24-week global study. Linagliptin was effective in improving glucose control in patients aged $\geq 60$ years on stable glucose-lowering therapy with basal insulin. Linagliptin was well tolerated and no new safety concerns were raised.

\section{ACKNOWLEDGEMENTS}

The authors thank the participants and staff involved in this study.

Funding. This study and the journal's Rapid Service fee and Open Access fee were funded by the Boehringer Ingelheim \& Eli Lilly and Company Diabetes Alliance. All authors had full access to all of the data in this study and take complete responsibility for the integrity of the data and accuracy of the data analysis.

Medical Writing Assistance. Medical writing assistance in the preparation of this manuscript was provided by Paul Nistor and Charlie Bellinger of Envision Scientific Solutions. Support for this assistance was funded by Nippon Boehringer Ingelheim Co., Ltd., and Eli Lilly Japan K.K.

Authorship. All named authors meet the International Committee of Medical Journal Editors (ICMJE) criteria for authorship for this article, were fully responsible for all content and editorial decisions, take responsibility for the integrity of the work as a whole and have given their approval for this version to be published.

Authorship Contributions. Substantial contributions to conception and design: Eiichi Araki, Yuko Tanaka and Wataru Sakamoto; substantial contributions to data acquisition, analysis and interpretation: Eiichi Araki, Yuriko Unno, Yuko Tanaka, Wataru Sakamoto and Yuki Miyamoto; statistical expertise: Wataru
Sakamoto; drafting the manuscript, revising for important intellectual content and approval of the final version: Eiichi Araki, Yuriko Unno, Yuko Tanaka, Wataru Sakamoto and Yuki Miyamoto.

Disclosures. Eiichi Araki has participated on advisory panels for Alcon, Astellas Pharma, Astra Zeneca, Eli Lilly, Kowa Pharmaceutical, Nippon Boehringer Ingelheim, Novo Nordisk Pharma, Sanofi and Terumo Corp., has received honoraria for lectures from Astellas Pharma, MSD, Ono Pharmaceutical, Novo Nordisk Pharma and Sanofi, and scholarship grants from Astellas Pharma, Daiichi Sankyo, Mitsubishi Tanabe Pharma, Nippon Boehringer Ingelheim, Novo Nordisk Pharma, Ono Pharmaceutical, Sanofi, Shionogi, Sumitomo Dainippon Pharma and Takeda Pharmaceutical; Yuriko Unno was an employee of Nippon Boehringer Ingelheim at the time of this study. Yuko Tanaka is an employee of Nippon Boehringer Ingelheim. Wataru Sakamoto is an employee of Nippon Boehringer Ingelheim.Yuki Miyamoto was an employee of Nippon Boehringer Ingelheim at the time of this study.

Compliance with Ethics Guidelines. The trial protocol was reviewed by the Independent Ethics Committees and/or Institutional Review Boards of the trial participating study sites (Supplementary Table 5). The study was conducted in accordance with the principles of the Declaration of Helsinki, the ICH GCP, the Japanese GCP regulations and the GPSP Good Post-Marketing Study Practice. All patients provided written informed consent.

Data Availability. To ensure independent interpretation of clinical study results, Boehringer Ingelheim grants all external authors access to all relevant material, including participant-level clinical study data and relevant material as needed by them to fulfill their role and obligations as authors under the ICMJE criteria. Furthermore, clinical study documents (e.g., study report, study protocol, statistical analysis plan) and participant clinical study data are available to be shared after publication of the primary manuscript in a peer-reviewed 
journal and if regulatory activities are complete and other criteria met per the BI Policy on Transparency and Publication of Clinical Study Data: https://trials.boehringer-ingelheim.com/ transparency_policy.html. Prior to providing access, documents will be examined, and, if necessary, redacted and the data will be deidentified to protect the personal data of study participants and personnel and to respect the boundaries of the informed consent of the study participants. Clinical Study Reports and Related Clinical Documents can be requested via this link: https://trials.boehringer-ingelheim. com/trial_results/clinical_submission_documents. html. All such requests will be governed by a Document Sharing Agreement. Bona fide, qualified scientific and medical researchers may request access to de-identified, analysable participant clinical study data with corresponding documentation describing the structure and content of the data sets. Upon approval, and governed by a Data Sharing Agreement, data are shared in a secured data-access system for a limited period of 1 year, which may be extended upon request. Researchers should use https:// clinicalstudydatarequest.com to request access to study data.

Open Access. This article is distributed under the terms of the Creative Commons Attribution-NonCommercial 4.0 International License (http://creativecommons.org/licenses/ by-nc/4.0/), which permits any noncommercial use, distribution, and reproduction in any medium, provided you give appropriate credit to the original author(s) and the source, provide a link to the Creative Commons license, and indicate if changes were made.

\section{REFERENCES}

1. Yabe D, Seino Y, Fukushima M, Seino S. Beta cell dysfunction versus insulin resistance in the pathogenesis of type 2 diabetes in East Asians. Curr Diab Rep. 2015;15(6):602.

2. International Diabetes Federation. IDF Diabetes Atlas, 8th edn. Brussels, Belgium. 2017. https:// www.diabetesatlas.org. Accessed 17 Nov 2017.
3. Seino Y, Kurahachi H, Goto Y, Taminato T, Ikeda M, Imura H. Comparative insulinogenic effects of glucose, arginine and glucagon in patients with diabetes mellitus, endocrine disorders and liver disease. Acta Diabetol Lat. 1975;12(2):89-99.

4. Seino Y, Kuwata H, Yabe D. Incretin-based drugs for type 2 diabetes: focus on East Asian perspectives. J Diabetes Investig. 2016;7(Suppl 1):102-9.

5. Japan Diabetes Society. Treatment guide for diabetes 2016-2017. http://www.fa.kyorin.co.jp/jds/ uploads/Treatment_Guide_for_Diabetes_2016-2017. pdf. Accessed 23 April 2018.

6. Report Committee. Glycemic targets for elderly patients with diabetes: Japan Diabetes Society (JDS)/Japan Geriatrics Society (JGS) Joint Committee on Improving Care for Elderly Patients with Diabetes. J Diabetes Investig. 2017;8(1):126-8.

7. Araki E, Haneda M, Kasuga M, et al. New glycemic targets for patients with diabetes from the Japan Diabetes Society. J Diabetes Investig. 2017;8(1):123-5.

8. Haneda $M$, Noda $M$, Origasa $H$, et al. Japanese clinical practice guideline for diabetes 2016. J Diabetes Investig. 2018;9(3):657-97.

9. Kim YG, Hahn S, Oh TJ, Kwak SH, Park KS, Cho YM. Differences in the glucose-lowering efficacy of dipeptidyl peptidase- 4 inhibitors between Asians and non-Asians: a systematic review and metaanalysis. Diabetologia. 2013;56(4):696-708.

10. Schernthaner G, Barnett AH, Emser A, et al. Safety and tolerability of linagliptin: a pooled analysis of data from randomized controlled trials in 3572 patients with type 2 diabetes mellitus. Diabetes Obes Metab. 2012;14(5):470-8.

11. Lehrke M, Marx N, Patel S, et al. Safety and tolerability of linagliptin in patients with type 2 diabetes: a comprehensive pooled analysis of 22 placebocontrolled studies. Clin Ther. 2014;36(8):1130-46.

12. Kawamori R, Inagaki N, Araki E, et al. Linagliptin monotherapy provides superior glycaemic control versus placebo or voglibose with comparable safety in Japanese patients with type 2 diabetes: a randomized, placebo and active comparator-controlled, double-blind study. Diabetes Obes Metab. 2012;14(4):348-57.

13. Inagaki $\mathrm{N}$, Watada $\mathrm{H}$, Murai $\mathrm{M}$, et al. Linagliptin provides effective, well-tolerated add-on therapy to pre-existing oral antidiabetic therapy over 1 year in Japanese patients with type 2 diabetes. Diabetes Obes Metab. 2013;15(9):833-43.

14. Araki E, Kawamori R, Inagaki N, et al. Long-term safety of linagliptin monotherapy in Japanese 
patients with type 2 diabetes. Diabetes Obes Metab. 2013;15(4):364-71.

15. Tradjenta [US prescribing information]. Boehringer Ingelheim. 2016. http://docs.boehringer-ingelheim. com/Prescribing\%20Information/PIs/Tradjenta/Tra djenta.pdf. Accessed 23 Jan 2017.

16. Trajenta [Japan prescribing information]. Japan Boehringer Ingelheim Co., Ltd./Japan Eli Lilly Co., Ltd. 2018. http://www.pmda.go.jp/PmdaSearch/ iyakuDetail/GeneralList/3969014F1. Accessed 3 April 2018.

17. Iwamoto Y, Kashiwagi A, Yamada N, et al. Efficacy and safety of vildagliptin and voglibose in Japanese patients with type 2 diabetes: a 12-week, randomized, double-blind, active-controlled study. Diabetes Obes Metab. 2010;12(8):700-8.

18. Iwamoto Y, Tajima N, Kadowaki T, et al. Efficacy and safety of sitagliptin monotherapy compared with voglibose in Japanese patients with type 2 diabetes: a randomized, double-blind trial. Diabetes Obes Metab. 2010;12(7):613-22.

19. Kadowaki T, Kondo K, Sasaki N, et al. Efficacy and safety of teneligliptin add-on to insulin monotherapy in Japanese patients with type 2 diabetes mellitus: a 16-week, randomized, double-blind, placebo-controlled trial with an open-label period. Expert Opin Pharmacother. 2017;18(13):1291-300.

20. Seino Y, Fujita T, Hiroi S, Hirayama M, Kaku K. Efficacy and safety of alogliptin in Japanese patients with type 2 diabetes mellitus: a randomized, double-blind, dose-ranging comparison with placebo, followed by a long-term extension study. Curr Med Res Opin. 2011;27(9):1781-92.

21. Fukuda M, Doi K, Sugawara M, Mochizuki K. Efficacy and safety of sitagliptin in elderly patients with type 2 diabetes mellitus: A focus on hypoglycemia. J Diabetes Investig. 2019;10(2):383-91.

22. Kadowaki T, Muto S, Ouchi Y, Shimazaki R, Seino Y. Efficacy and safety of saxagliptin in combination with insulin in Japanese patients with type 2 diabetes mellitus: a 16-week double-blind randomized controlled trial with a 36-week open-label extension. Expert Opin Pharmacother. 2017;18(18):1903-19.

23. Saito D, Kanazawa A, Shigihara N, et al. Efficacy and safety of vildagliptin as an add-on therapy in inadequately controlled type 2 diabetes patients treated with basal insulin. J Clin Med Res. 2017;9(3):193-9.

24. McGill JB, Yki-Jarvinen H, Crowe S, Woerle HJ, von Eynatten M. Combination of the dipeptidyl peptidase-4 inhibitor linagliptin with insulin-based regimens in type 2 diabetes and chronic kidney disease. Diab Vasc Dis Res. 2015;12(4):249-57.

25. Ledesma G, Umpierrez GE, Morley JE, et al. Efficacy and safety of linagliptin to improve glucose control in older people with type 2 diabetes on stable insulin therapy: a randomized trial. Diabetes Obes Metab. 2019. https://doi.org/10.1111/dom.13829 [Epub ahead of print].

26. PMDA. Basic principles on global clinical trials. 2007. https://www.pmda.go.jp/files/000153265. pdf. Accessed 27 April 2018.

27. American Diabetes Association. 6. Glycemic targets: standards of medical care in diabetes-2018. Diabetes Care. 2018;41(Suppl 1):S55-S64.

28. Schernthaner G, Barnett AH, Patel S, Hehnke U, von Eynatten M, Woerle HJ. Safety and efficacy of the dipeptidyl peptidase-4 inhibitor linagliptin in elderly patients with type 2 diabetes: a comprehensive analysis of data from 1331 individuals aged $>/=65$ years. Diabetes Obes Metab. 2014;16(11):1078-86.

29. Nauck M, Araki A, Hehnke U, Plat A, Clark D, Khunti K. Risk of hypoglycaemia in people aged $\geq 65$ years receiving linagliptin: pooled data from 1489 individuals with type 2 diabetes mellitus. Int J Clin Pract. 2018;72(10):e13240.

30. Kaku K, Sumino S, Katou M, Nishiyama Y, Kinugawa Y. Randomized, double-blind, phase III study to evaluate the efficacy and safety of once-daily treatment with alogliptin and metformin hydrochloride in Japanese patients with type 2 diabetes. Diabetes Obes Metab. 2017;19(3):463-7.

31. Inagaki N, Onouchi H, Sano H, Funao N, Kuroda S, Kaku K. SYR-472, a novel once-weekly dipeptidyl peptidase-4 (DPP-4) inhibitor, in type 2 diabetes mellitus: a phase 2, randomised, double-blind, placebo-controlled trial. Lancet Diabetes Endocrinol. 2014;2(2):125-32.

32. Araki E, Inagaki N, Tanizawa Y, Oura T, Takeuchi M, Imaoka T. Efficacy and safety of once-weekly dulaglutide in combination with sulphonylurea and/or biguanide compared with once-daily insulin glargine in Japanese patients with type 2 diabetes: a randomized, open-label, phase III, non-inferiority study. Diabetes Obes Metab. 2015;17(10):994-1002.

33. Nino A, Okuda I, Wilson TH, et al. Weekly glucagon-like peptide-1 receptor agonist albiglutide as monotherapy improves glycemic parameters in Japanese patients with type 2 diabetes mellitus: a randomized, double-blind, placebo-controlled study. J Diabetes Investig. 2018;9(3):558-66. 